\title{
EARTH OBSERVATION SYSTEM PLANS OF INDIA
}

\author{
M.G. CHANDRASHEKAR, V. JAYARAMN, C.B.S. DUTT, B. MANIKIAM \\ Indian Space Research Organisation (Headquarters), Bangalore, India
}

\begin{abstract}
Operational methodologies are available to retrieve several parameters related to the land, air and oceans from satellite data which is capable of providing well calibrated $\mathrm{data} /$ observations over large areas giving a synoptic view on a repetitive and reliable basis. The capability of satellites to provide data in various spectral, spatial and temporal scales is of great advantage in studying the dynamic aspects of earth atmosphere system. The present day capabilities of satellites include spatial resolutions ranging from $10 \mathrm{~m}$ and above and repetition of a few hours (geosynchronous Satellite) to few days. Higher spatial resolutions and all weather capabilities (through microwave sensing) are becoming available in the immediate future.Towards utilising the potentials of space based systems, India has been operating INSAT series of satellite for weather monitoring and IRS series of satellites for natural resources monitoring/management. The INSAT is a series of geostationary satellites stationed over Indian region to provide meteorological observations on a continuous basis in visible and thermal regions in addition to providing services for disaster warning related to Cyclones and remote location data collection platforms. The space based observations on meteorology over the past 5 years is proving to be a valuable data base for studies related to monsoon dynamics and tropical cyclones.

The IRS series of satellites are planned to provide Remote Sensing Observations to cater to the requirements of natural resources monitoring and management. The high resolution data obtained from IRS-IA presently is being complimented by data from contemporary satellites such as Landsat, SPOT and NOAA. A large number of operational packages have been developed to suit the user needs. A National Natural Resources Management System has been established in the country with thrust on use of remote sensing for management of resources in the country. In future, it is planned to have Remote Sensing satellite with enhanced capabilities by way of better resolutions, more spectral bands and stereo capabilities. Development of Microwave sensor on air-craft based platform is in progress towards reaching satellite payload capabilities in near future.
\end{abstract}

Y. Kondo (ed.), Observatories in Earth Orbit and Beyond, 531.

(C)1990 Kluwer Academic Publishers. Printed in The Netherlands. 